\title{
The Path To Visible Extreme Adaptive Optics With MagAO-2K and MagAO-X
}

Jared R. Males ${ }^{1}$, Laird M. Close ${ }^{1}$, Olivier Guyon ${ }^{1,2}$, Katie M. Morzinski ${ }^{1}$, Philip Hinz ${ }^{1}$, Simone Esposito $^{3}$, Enrico Pinna ${ }^{3}$, Marco Xompero ${ }^{3}$, Runa Briguglio ${ }^{3}$, Armando Riccardi ${ }^{3}$, Alfio Puglisi $^{3}$, Ben Mazin ${ }^{4}$, Michael J. Ireland ${ }^{5}$, Alycia Weinberger ${ }^{6}$, Al Conrad ${ }^{1}$, Matthew Kenworthy $^{7}$, Frans Snik ${ }^{7}$, Gilles Otten ${ }^{7}$, Nemanja Jovanovic ${ }^{2}$, and Julien Lozi ${ }^{2}$

${ }^{1}$ Steward Observatory, University of Arizona, Tucson AZ 85721, USA

${ }^{2}$ Subaru Telescope, National Astronomical Observatory of Japan, Hilo, HI, 96720, USA.

${ }^{3} \mathrm{INAF}$, Osservatorio Astrofisico di Arcetri, Firenze, Italy

${ }^{4}$ University of California Santa Barbara, Santa Barbara CA, USA

${ }^{5}$ Research School of Astronomy and Astrophysics, Australian National University, Canberra, ACT 2611, Australia

${ }^{6}$ Department of Terrestrial Magnetism, Carnegie Institute of Washington, Washington, DC 20015, USA

${ }^{7}$ Leiden Observatory, Leiden University, P.O. Box 9513, 2300 RA Leiden, The Netherlands

\begin{abstract}
The next generation of extremely large telescopes (ELTs) have the potential to image habitable rocky planets, if suitably optimized. This will require the development of fast high order "extreme" adaptive optics systems for the ELTs. Located near the excellent site of the future GMT, the Magellan AO system (MagAO) is an ideal on-sky testbed for high contrast imaging development. Here we discuss planned upgrades to MagAO. These include improvements in WFS sampling (enabling correction of more modes) and an increase in speed to $2000 \mathrm{~Hz}$, as well as an H2RG detector upgrade for the Clio infrared camera. This NSF funded project, MagAO-2K, is planned to be on-sky in November 2016 and will significantly improve the performance of MagAO at short wavelengths. Finally, we describe MagAO-X, a visible-wavelength extreme-AO "afterburner" system under development. MagAO-X will deliver Strehl ratios of over $80 \%$ in the optical and is optimized for visible light coronagraphy.
\end{abstract}

Keywords: adaptive optics, exoplanets, high contrast imaging

\section{INTRODUCTION}

Direct imaging of exoplanets has produced many spectacular results. Recent examples include the discovery and characterization of 51 Eri b, ${ }^{1}$ the detection of accretion on LkCa $15 \mathrm{~b},{ }^{2}$ and the fascinating discovery of HD 131399Ab. ${ }^{3}$ Yet, of the 3,302 confirmed planets and $>4700$ candidates*, only a handful have been imaged and spectro-photometrically characterized. Direct imaging is, in some sense, in its infancy. Continued effort is needed to further develop our direct imaging capabilities, especially as we prepare for the revolutionary advances enabled by the next generation of extremely large telescopes (ELTs). ${ }^{4,5}$

Here we describe work underway and planned for the Magellan Adaptive Optics System (MagAO) ${ }^{6,7}$ to upgrade its performance and develop new capabilities. The main focus of these efforts is imaging exoplanets, ${ }^{8}$ particularly at visible wavelengths. ${ }^{9}$ We first describe the MagAO-2K project, an NSF/ATI funded upgrade of the existing MagAO system. We then present the rationale and conceptual design of MagAO-X, a planned visible-wavelength coronagraph extreme AO (ExAO) system.

Further author information: (Send correspondence to J.R.M.)

J.R.M.: E-mail: jrmales@as.arizona.edu

*As of July 2016; using http://exoplanetarchive.ipac.caltech.edu/ and http://kepler.nasa.gov/ 


\section{UPGRADING MAGAO TO A 2 KHZ EXTREME-AO SYSTEM}

The goal of the first phase of planned upgrades to MagAO is to increase speed to $2000 \mathrm{~Hz}$, control 450 modes, and reduce residual jitter from 8 mas to 4 mas. Here we describe this upgrade, and present a summary of our performance analysis and simulations of the improved system.

\subsection{Modeling MagAO at LCO}

LCO Atmosphere Model: We used a model of the LCO atmosphere based on the GMT site survey ${ }^{10}$ and LCO seeing statistics and outer scale $L_{0}=20 \mathrm{~m} .{ }^{11}$ We use only DIMM seeing to have a valid measure of $r_{0}$ independent of $L_{0}$. In our end-to-end simulations we include the outer scale (von Kármán statistics) and the median $C_{n}^{2}$ profile. For the anaTable 1. Summary of MagAO Atmosphere Models

\begin{tabular}{|c|c|c|c|c|}
\hline \multirow[b]{2}{*}{ Quartile } & \multirow[b]{2}{*}{$\begin{array}{c}\bar{v} \\
{[\mathrm{~m} / \mathrm{s}]}\end{array}$} & \multicolumn{3}{|c|}{ @ $V(0.55 \mu \mathrm{m})$} \\
\hline & & $\begin{array}{c}\text { FWHM } \\
{["]}\end{array}$ & $\begin{array}{c}r_{0} \\
{[\mathrm{~m}]}\end{array}$ & $\begin{array}{c}\tau_{0} \\
{[\mathrm{msec}]}\end{array}$ \\
\hline $25 \%$ & 9.4 & 0.51 & 0.22 & 7.4 \\
\hline $50 \%$ & 18.7 & 0.65 & 0.17 & 2.9 \\
\hline $75 \%$ & 23.4 & 0.81 & 0.14 & 1.9 \\
\hline
\end{tabular}

lytic error budget we use quantities averaged over these layers (cf. sect. 3.3.6 of Hardy, $1998^{12}$ ). Our end-to-end simulations include multiple layers using the $C_{n}^{2}$ profile from site testing. ${ }^{10}$

We normally have little knowledge of wind speed at altitude. We incorporate our own experience at LCO (166 nights on-sky with MagAO) to estimate typical winds. As a baseline we use the GMT survey wind layers, which have a $C_{n}^{2}$ weighted mean of $\bar{v}=18.7 \mathrm{~m} / \mathrm{s}$. To model good conditions, we use winds $50 \%$ slower. To model bad conditions, we use winds $25 \%$ faster.

We summarize our atmosphere model in Table 1. For this analysis we use 3 distinct models, intended to represent "excellent", "good", and "fair" conditions. The "25\%" model corresponds to first quartile DIMM seeing, and low winds. The " $50 \%$ " model corresponds to median DIMM seeing, and the GMT atmosphere winds. The "75\%" model includes third quartile DIMM seeing, and higher winds. Wind and seeing are not necessarily coupled, so various combinations of these parameters are possible.

Analytic Error Budgets: To evaluate the performance improvements we first analyzed our system error budget using mostly standard relationships valid for Kolmogorov
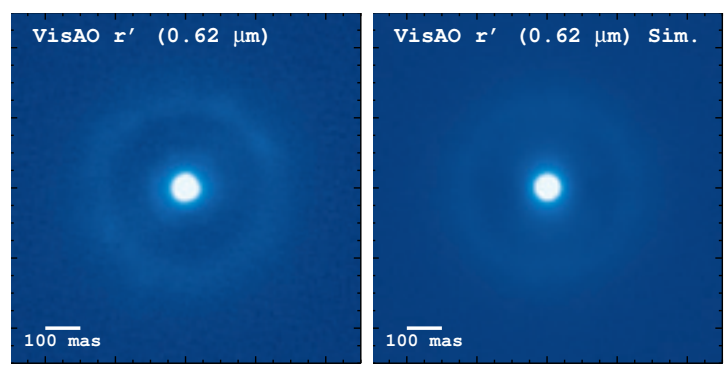

Figure 1. MagAO $r^{\prime}$ PSF on-sky (left) compared to simulation (right) in similar conditions. This validates our atmosphere model, vibration model, and end-toend simulations.

turbulence. We use a fitting error relationship which was calculated specifically for the MagAO Karhunen-Loève (KL) basis set. ${ }^{13}$ Pyramid wavefront sensor (PyWFS) sensitivity vs. signal-to-noise ratio (S/N) was estimated using the relationships developed in Guyon (2005). ${ }^{14}$ For S/N we use the WFS bandpass, including the CCD QE curve, dichroics and beamsplitters. The MagAO-WFS filter curve was then used to integrate the HST calspec Vega spectrum. ${ }^{15}$ We also included static wavefront error (WFE) of $50 \mathrm{~nm}$ rms phase, based on post-integration shell flattening, and non-common path (NCP) WFE of $30 \mathrm{~nm}$ rms phase measured on the PyWFS beamsplitters.

End-to-End Simulations: We also used end-to-end simulations with the CAOS IDL package, ${ }^{16}$ which has been used extensively throughout the MagAO project. ${ }^{13,17}$ Fig. 1 compares an on-sky measurement of the $r^{\prime}$ $(0.62 \mu \mathrm{m})$ PSF with a simulation for similar conditions, jitter, and static and NCP WFE, validating our CAOS simulations.

Jitter: We model the impact of residual jitter on SR by convolving a Gaussian having a width equal to the rms jitter with a Gaussian of FWHM $=\lambda / D$. This yields Eqn. 1 where $\sigma_{t / t}$ is the rms jitter in the same units as $\lambda / D$, and $\kappa=0.98$ accounts for the reduction in FWHM due to the $29 \%$ central obscuration.

$$
S_{t / t}=\left[1+\frac{8 \ln (2)}{\kappa^{2}}\left(\frac{\sigma_{t / t}}{\lambda / D}\right)^{2}\right]^{-1}
$$




\subsection{Harnessing MagAO's Spatial Degrees of Freedom}

Two components can set the upper limit on controllable degrees of freedom (DOF), or modes. With a $29 \%$ central obscuration, MagAO has 540 illuminated actuators. This sets the absolute upper limit on DOF at 540. With the current 27 pixels across PyWFS pupils, there are 508 illuminated sub-apertures, which in principle sets a further limit on controllable DOF. In operation, MagAO is currently limited to only 300 stably controlled modes. There are two straightforward steps we will take to maximize MagAO's usable DOF.

\subsubsection{Recalibration}

The first step is to re-calibrate the ASM capacitive position sensors. The procedure for performing this calibration is described in detail in Esposito (2010). ${ }^{18}$ In brief, a Twyman-Green interferometer is used in double pass to measure the shape of the ASM. The interferometer laser is returned by a retroreflector rigidly suspended at the $f / 1$ focus $\sim 1 \mathrm{~m}$ below the ASM by a carbon fiber truss. This procedure was used to calibrate MagAO in the lab (Fig. 2), and it is now routinely used with the nearly identical AO systems at the LBT.

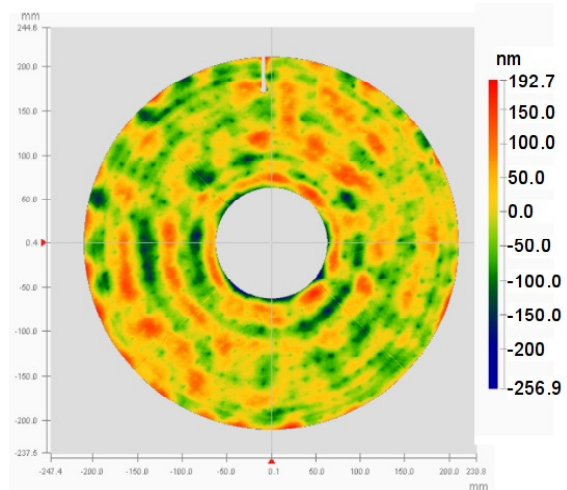

Figure 2. Phase map of the MagAO ASM flat measured using the $4 \mathrm{D}$ in the Arcetri test tower lab (50 $\mathrm{nm}$ rms phase residual). We plan to perform this measurement at $\mathrm{LCO}$ in November, 2016.
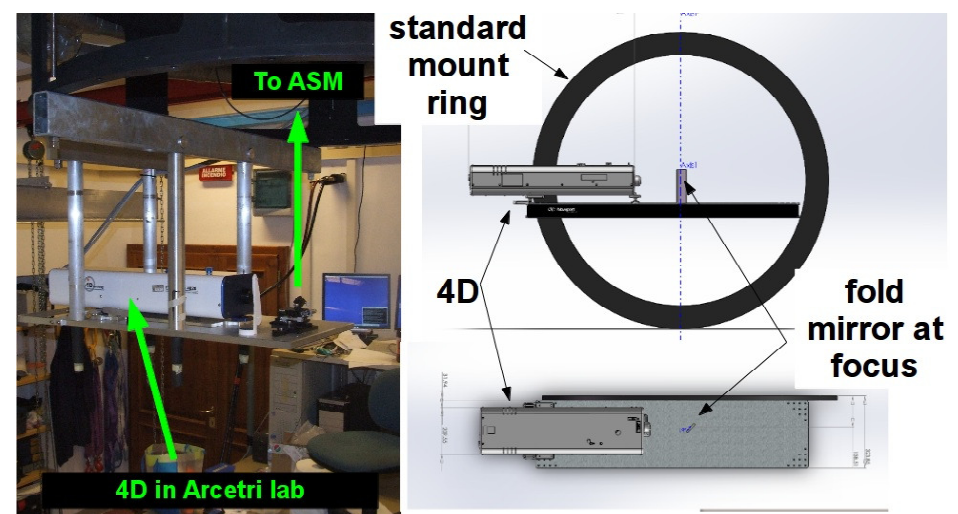

Figure 3. Left: The 4D interferometer in use at the Arcetri test-tower lab. Right: Solid model showing the notional layout of the 4D and the mounting ring.

The MagAO ASM has not been calibrated interferometrically since integration at Arcetri, Italy, in 2011. We have measured interaction matrices at LCO with the retroreflector, but without a recent calibration of the capacitive sensors we are currently using a sub-optimal basis set on-sky. This limits stable operations to 300 modes in median conditions. In $25 \%$ conditions the system controls 378 modes. To re-calibrate the system, a 4D PhaseCam 4020 interferometer (hereafter the 4D) will temporarily replace Clio2. MagAO will be able to control at least 400 modes in median conditions after recalibration, based on the lab testing of MagAO (with a fresh calibration), current performance, and results achieved at the LBT.

\subsubsection{WFS Re-Sampling}

The sampling of the PyWFS pupils on the CCD is set by the pupil relay lens, and is currently 27 pixels across the pupil. 508 pixels within this area are included in the slope calculation, and even though this nominally provides 508 DOF, the loop becomes unstable if we try to control more than $\sim 425$ modes in simulations. Increasing sampling to 30 pixel diameter will allow us to control up to 450 modes. Re-sampling will be accomplished by changing the magnification of the pupil relay lens after the pyramid. An important consideration in increasing the number of pixels is the impact on latency during slope computation, communication, and reconstruction. We analyze this below when discussing servo-lag at various loop speeds. 


\subsection{Achieving $2000 \mathrm{~Hz}$}

Here we describe how the speed of MagAO can be increased to between $1.5 \mathrm{kHz}$ (baseline) and $2.0 \mathrm{kHz}$ (goal).

\subsubsection{Reducing ASM Response Time}

The ASM electronics are capable of accepting commands at 1800 $\mathrm{Hz}$, with potential to reach $2000 \mathrm{~Hz}$. This will allow us to run the loop at this speed, and provide an immediate improvement in response time. We are also considering modifications to the control firmware of the ASM. The Microgate company (Bolzano, Italy) has developed a new "dynamic feed-forward" matrix, which has been tested on the ASM for the VLT. If simply running the loop faster does not provide sufficient improvement in ASM response, we will implement this algorithm on the MagAO ASM. This would require only a software change: no hardware modifications are necessary.

Table 2. MagAO Servo Lag. All times in msec.

\begin{tabular}{|c|c|c|c|}
\hline & & \multicolumn{2}{|c|}{ Upgrade } \\
& Current & Baseline & Goal \\
\hline Speed & $1000 \mathrm{~Hz}$ & $1500 \mathrm{~Hz}$ & $2000 \mathrm{~Hz}$ \\
\hline$t_{\text {exp }}$ & 1.00 & 0.67 & 0.50 \\
$t_{f / t}$ & $\sim 0$ & $\sim 0$ & $\sim 0$ \\
$t_{r d}$ & 1.00 & 0.67 & 0.50 \\
$t_{\text {sl }}$ & 0.15 & 0.15 & 0.19 \\
$t_{\text {rec }}$ & 0.15 & 0.15 & 0.16 \\
$t_{\text {com }}$ & 0.04 & 0.04 & 0.06 \\
$t_{\text {settl }}$ & 1.0 & 0.67 & 0.5 \\
\hline$\Delta t$ & 2.84 & 2.02 & 1.66 \\
$\sigma_{\Delta t}[\mathrm{~nm}]$ & 78.2 & 62.5 & 48.2 \\
\hline
\end{tabular}

\subsubsection{Increasing PyWFS and Reconstruction Speed}

To run at $>1 \mathrm{kHz}$ we also need to increase the sampling rate of the PyWFS. The CCD-39, with Scimeasure Little Joe electronics, already runs at $2 \mathrm{kHz}$ with readout noise $(\mathrm{RON})$ increased from $\sim 8$ electrons to $\sim 12$ electrons. This RON increase only affects speeds $>1 \mathrm{kHz}$ and the slowest, most sensitive modes ( $\sim 3.5$ electrons) are unchanged, so this does not affect faint guide star science.

Though the CCD-39 clocks at $2 \mathrm{kHz}$, two components of the PyWFS will need to be upgraded. The first is the combination framegrabber and slope computer, called the BCU-39. Since the LBT PyWFS is 30 pixels across, the BCU-39 already supports our baseline upgrade to 30 pixels across at MagAO. Our plan to increase speed to between $1.5 \mathrm{kHz}$ to $2.0 \mathrm{kHz}$ requires hardware modifications to the BCU-39. An upgraded BCU-39 will be provided by Microgate.

The other PyWFS component which must be improved is the PI fast steering mirror, which modulates the beam around the pyramid tip. Our PI 300.4SL tip-tilt head can reach $2 \mathrm{kHz}$ with our lightweight $12 \mathrm{~mm} \times 3$ $\mathrm{mm}$ mirror, but the Microgate driver electronics must be upgraded to support operations at $2 \mathrm{kHz}$. The new driver electronics will also be provided by Microgate.

\subsubsection{Servo-lag}

The improvement in speed just described will impact MagAO's error budget primarily in the servo-lag component. Because of the finite loop speed, once a wavefront measurement is applied to the ASM it is out of date as turbulence is blown down wind. We denote the residual wavefront error due to this servo-lag as $\sigma_{\Delta t}$. In our model of MagAO we include the following latencies: exposure time $t_{\text {exp }}$; frame transfer time $t_{f / t}(\sim 0)$; readout time $t_{r d}\left(=t_{\text {exp }}\right)$; slope computation $t_{s l}$; reconstruction $t_{r e c}$; time for communications between the slope computer, reconstructor, and ASM, $t_{\text {com }}$; and settling time $t_{\text {settl }}$. Total latency is given by Equation 2 . We treat the midpoint of the CCD-39 exposure as the measurement time. Values of these delays are shown in Table 2, along with the baseline $(1.5 \mathrm{kHz})$ and goal $(2.0 \mathrm{kHz})$ upgrade values. We also show $\sigma_{\Delta t}$ for $50 \%$ conditions.

$$
\Delta t=\frac{1}{2} t_{e x p}+t_{f / t}+t_{r d}+t_{s l}+t_{r e c}+t_{c o m}+t_{s e t t l}
$$

Our baseline upgrade to 30 pixels is within the current capacity of all hardware due to its LBT heritage, so no increase in $t_{s l}+t_{r e c}+t_{c o m}$ will occur. 

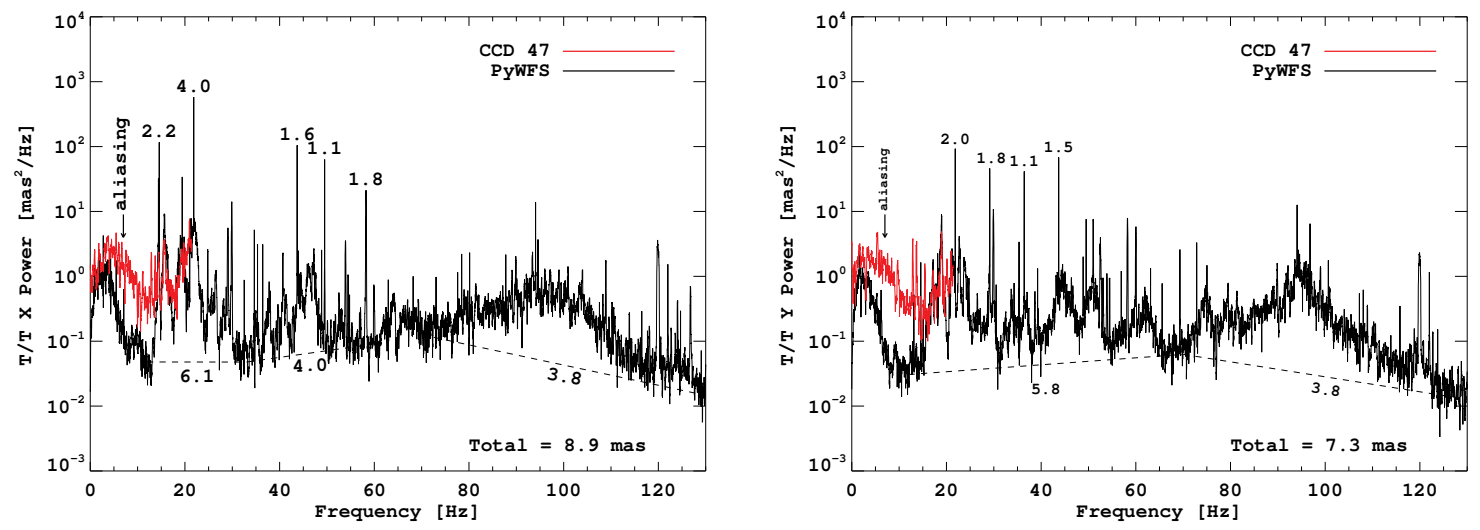

Figure 4. Vibration PSDs measured in $1 \mathrm{kHz}$ closed-loop on-sky. The CCD-47 image position was measured at 42 f.p.s., and the PyWFS tip and tilt modal amplitudes were recorded at $333 \mathrm{~Hz}$ (1/3 loop speed). The PyWFS tip/tilt PSDs (black) were normalized to the CCD-47 PSDs (red) from 15 to $21 \mathrm{~Hz}$ (lower frequencies are subject to aliasing). Peaks are labeled with root-integrated-power in mas. The area between the PSD and a simple estimated continuum is also indicated. Also see Garcés et $\mathrm{al}^{20}$ and Fernández et $a{ }^{21}{ }^{21}$ in these proceedings.

\subsection{Vibrations}

MagAO is subject to Strehl loss due to vibrations, as are all AO systems. ${ }^{19}$ To quantify this we simultaneously recorded VisAO focal-plane data at 42 frames per second (f.p.s.) and PyWFS reconstructed tip and tilt (T/T) modal amplitudes at $1 / 3$ loop speed, in closed-loop at $1 \mathrm{kHz}$. We then calculate the power spectral density (PSD) of the $\mathrm{x}$ and $\mathrm{y}$ positions, normalized to the rms position in each dimension. The T/T PSDs were calculated similarly and normalized to the CCD-47 between 15 and $21 \mathrm{~Hz}$. The result of these measurements is shown in Fig 4 where we label the root integrated power in narrow lines, as well as between the PSD and a simple continuum estimate indicated by the dashed lines. The total integral of the T/T PSD is indicated for each axis. We use the average ( 8.1 mas rms) as the current value of jitter. Vibration amplitudes add in quadrature, so removing a 4.0 mas peak would reduce $\mathrm{x}$-axis vibrations from 8.9 to 8.0 mas.

We have already made some efforts to quiet the environment of MagAO. The results shown in Fig 4 are after we reduced mean jitter from 12-15 mas rms to 8.1 mas rms. For instance, we have redesigned the CCD cooling system to use telescope glycol instead of a local pump. We have made significant changes to our offloading strategy - the process by which excess low-order modes are sent to the telescope - to minimize the excitation of structural vibrations. Work to identify and mitigate vibration sources continues (see Garcés et al ${ }^{20}$ and Fernández et al, ${ }^{21}$ in these proceedings).

Some decrease in jitter will result from increased speed: increasing speed to $1.5 \mathrm{kHz}$ reduces jitter to 7.0 mas rms. At $\mathrm{H} \alpha(0.656 \mu \mathrm{m})$ this is a $13 \%$ relative increase in Strehl. However, increasing loop speed alone will not address discrete mechanical sources causing the narrow peaks. A well established control technique is the linear quadratic Gaussian (LQG) controller. ${ }^{22,23}$ Such a Kalman-filter based optimal controller has been developed for the LBT architecture, ${ }^{24}$ including the necessary reconstructor software. As part of MagAO-2K we will commission this software at MagAO, and develop procedures for optimally identifying vibration peaks and suppressing them with the LQG. We estimate the gain from a preliminary analysis of the power contained in discrete peaks and the fact that they are stable over $>10$ minutes.

In MagAO-2K, we will reduce jitter from 8.1 mas to 6.0 mas rms, including the increase in bandwidth and suppression of the two most stable narrow peaks using the LQG, and modest $\sim 2$ mas per axis reduction from mechanical vibration control. This will improve $S_{t / t}$ by $28 \%$ at $\mathrm{H} \alpha$. Our goal is 4.0 mas rms, which will require an additional reduction of $\sim 3.5$ mas $\mathrm{rms}$ improvement in each axis.

Table 3. MagAO-2K Vibrations.

\begin{tabular}{|l|c|c|}
\hline & $\sigma_{t / t}$ & $S_{t / t} @ \mathrm{H} \alpha$ \\
\hline Current & $8.1 \mathrm{mas}$ & 0.53 \\
Baseline & $6.0 \mathrm{mas}$ & 0.68 \\
Goal & $4.0 \mathrm{mas}$ & 0.82 \\
\hline
\end{tabular}

This ambitious goal will be pursued with a comprehensive vibration engineering effort at the telescope, including 
accelerometer surveys and continued equipment-on/off closed-loop testing. Achieving this goal would improve $S_{t / t}$ by $55 \%$ at $\mathrm{H} \alpha$. The planned vibration improvements are summarized in Table 3.

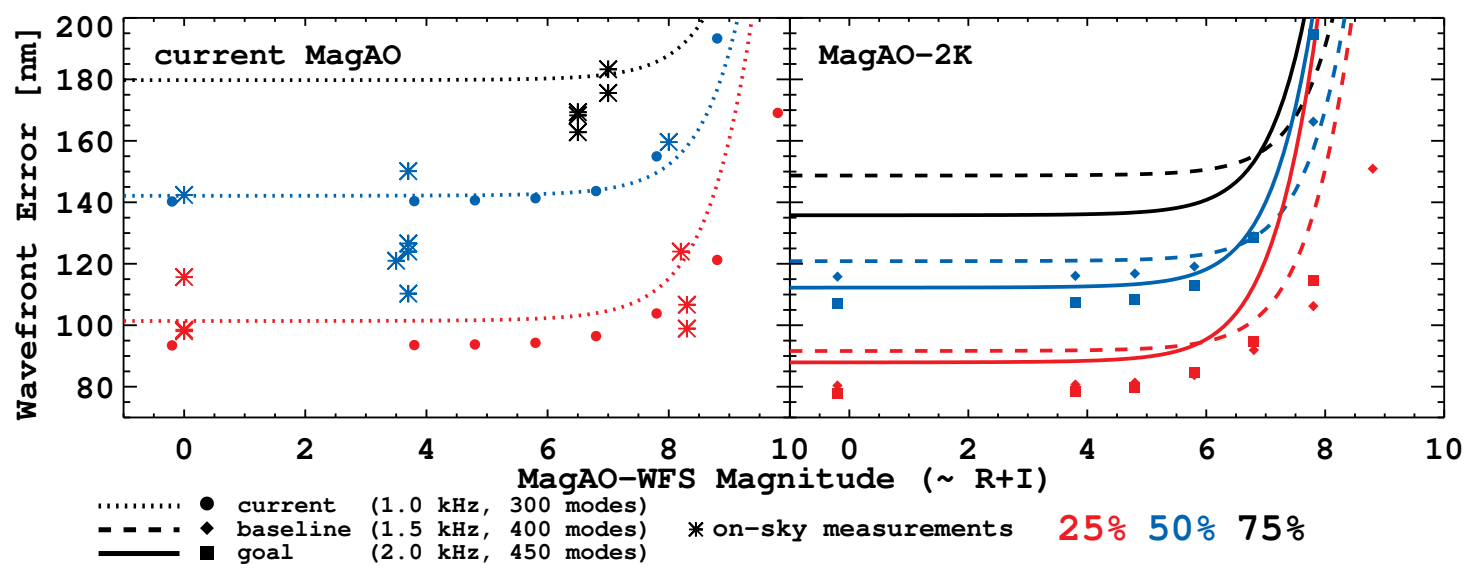

Figure 5. MagAO wavefront error (WFE). Left panel: current WFE, in 25\%-ile (red), 50\%-ile (blue), and 75\%-ile (black) conditions without jitter. Lines are the analytic error budget, filled symbols are end-to-end simulations (for $25 \%$ and $50 \%$ ), and asterisks are on-sky measurements from VisAO, corrected for 8.1 mas jitter. Our models of MagAO performance at LCO are validated by the on-sky data. Right panel: predicted performance of MagAO-2K under the same conditions. The baseline upgrade will produce performance in $75 \%$ conditions equal to current-system performance in $50 \%$ conditions.

\subsection{WFE Predictions}

The following table summarizes the MagAO-2K extreme-AO upgrade baseline and goal, compared to the current system. Total WFE is given for a 5 th magnitude guide star.

\begin{tabular}{lccccccc} 
& & & & \multicolumn{2}{c}{ Total WFE $[\mathrm{nm}]$} \\
& Speed & PyWFS Sampling & Controlled Modes & $25 \%$ & $50 \%$ & $75 \%$ & Jitter rms \\
\hline current & $1 \mathrm{kHz}$ & $27 \times 27$ & 300 modes & 102 & 142 & 180 & 8.1 mas \\
\hline baseline & $\mathbf{1 . 5} \mathbf{~ k H z}$ & $\mathbf{3 0 x 3 0}$ & $\mathbf{4 0 0}$ modes & $\mathbf{9 2}$ & $\mathbf{1 2 1}$ & $\mathbf{1 4 5}$ & $\mathbf{6 . 0}$ mas \\
goal & $\mathbf{2 . 0} \mathbf{~ k H z}$ & $\mathbf{3 0 x 3 0}$ & $\mathbf{4 5 0}$ modes & $\mathbf{9 0}$ & $\mathbf{1 1 4}$ & $\mathbf{1 3 7}$ & $\mathbf{4 . 0}$ mas
\end{tabular}

To concisely describe MagAO performance under various atmospheric conditions and system configurations, we present our error budget graphically. We compare our analytic error budget and simulations to on-sky measurements in the left panel of Fig 5. The on-sky measurements, taken in various conditions, have been corrected for 8.1 mas of jitter (Eqn. 1). The modeled results bound the on-sky measurements very well. The right-hand panel shows our predictions for the MagAO-2K upgrades with the same models. MagAO-2K will give the same performance in $\mathbf{7 5 \%}$ seeing and wind as currently obtained in $\mathbf{5 0 \%}$ conditions. The various parameters are somewhat independent of each other - we may achieve a mixture of the goal and baseline upgrades. Such a result will be bounded by the predictions we make here, and for clarity we do not present all permutations.

Due to the increased RON and decreased integration time, the limiting magnitude for $1.5 \mathrm{kHz}$ and $2.0 \mathrm{kHz}$ is brighter than for $1 \mathrm{kHz}$. We retain the ability to operate at $1 \mathrm{kHz}$, and all other lower speeds and PyWFS pixel binnings, so this does not impact guide star limiting magnitudes.

\subsection{Impact On AO Science}

Achieving these AO performance gains will provide dramatic benefits in all areas of VisAO science. To quantify this, we convert the WFE predictions to Strehl ratio in Fig 6 and include jitter. The grey shaded region corresponds to the current system between $50 \%$ and $25 \%$ conditions. The blue hatched region is the same, but for MagAO-2K baseline, and the orange region is for MagAO-2K goal. MagAO-2K will allow VisAO to operate in $75 \%$ conditions, when now we typically limit VisAO to $50 \%$ or better. 


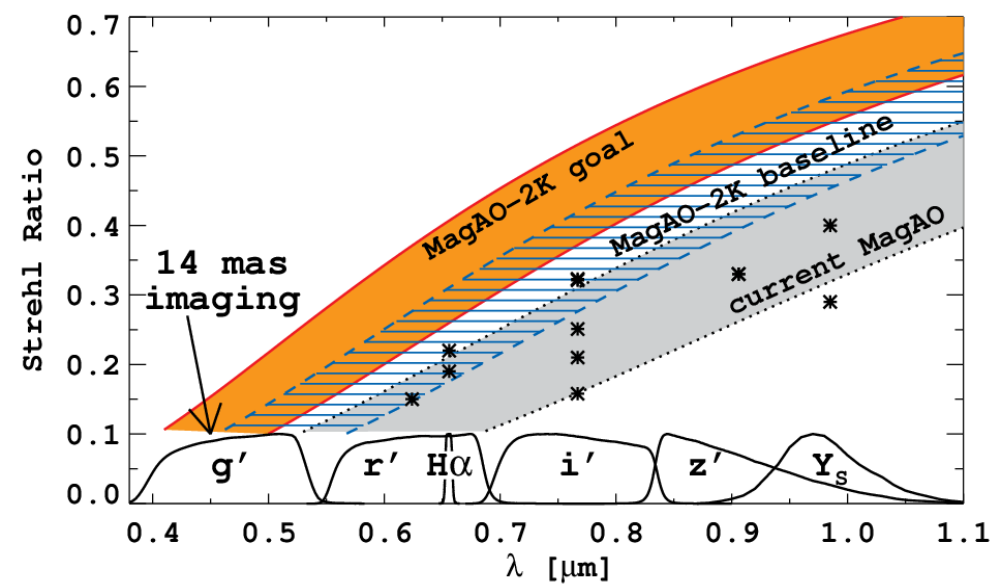

Figure 6. Predicted Strehl ratio after the MagAO-2K upgrade, compared to current system performance. The grey region corresponds to the current system operating in conditions ranging from $25 \%$ to $50 \%$, and the asterisks are on-sky measurements. The blue-hatched region is the baseline upgrade, and the orange region is the goal upgrade.

Science with Clio will benefit just as greatly. Lower WFE will benefit small inner working angle coronagraphy with Clio, and higher Strehl improves $S / N$ in the challenging background-limited thermal-IR. When coupled with the powerful H2RG detector upgrade (discussed below), the higher Strehl ratios will improve our already very competitive performance at $J, H$, and $K .^{25}$

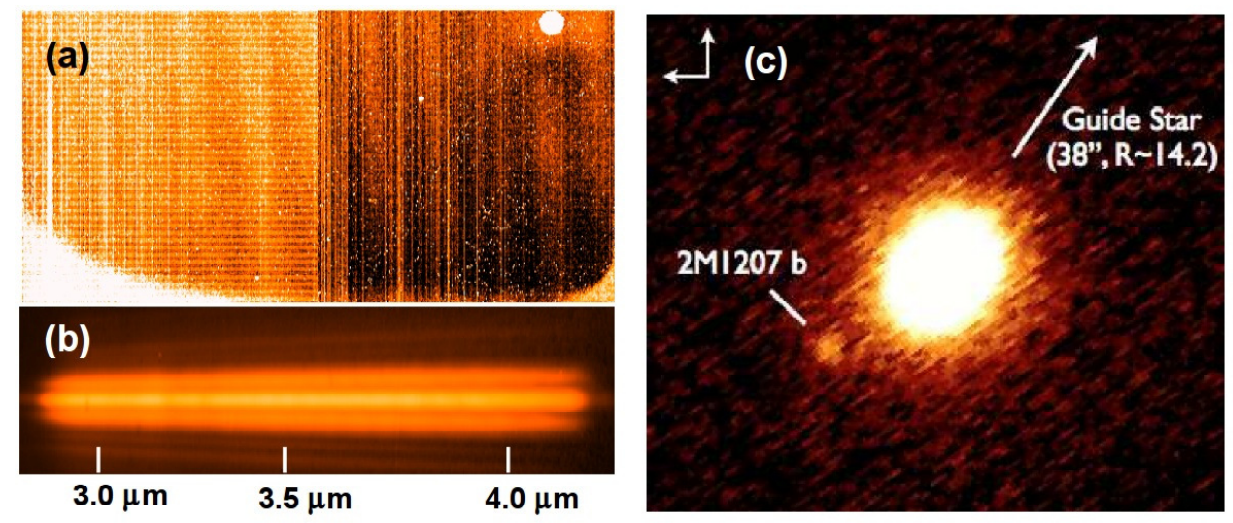

Figure 7. Clio2 on-sky. (a) A short exposure dark, showing numerous cosmetic defects and $>10 \%$ non-functional pixels which this upgrade will eliminate. (b) $L$-band spectrum of a young low-mass object with MagAO+Clio. ${ }^{26}$ (c) Brown dwarf $2 \mathrm{M} 1207,38$ " from a 14.2-mag guide star, with its 0.8 " planetary-mass companion in 1 " $V$ band seeing, at $\lambda=3.3$ $\mu \mathrm{m} . \mathrm{FWHM}=184$ mas. $^{27} \mathrm{MagAO}+$ Clio is ideal for this work due to the faint NGS off-axis capability.

\section{CLIO DETECTOR UPGRADE}

Clio is an imager and spectrometer which was developed specifically to detect cool exoplanets in the 3-5 $\mu \mathrm{m}$ atmospheric windows ${ }^{28,29}$ taking advantage of the thermally optimized ASM. It initially served as a general purpose high spatial resolution camera for the MMT AO system. Clio provides J through M filters, a prism spectrograph, and apodizing phase plate (APP) coronagraphs. In 2012 Clio was refurbished (now Clio2) and moved to LCO to serve as the first light IR camera for MagAO. In Figure 7 we show a dark, a spectrum, and a faint off-axis science result.

Clio2 currently has an "engineering grade" HAWAII-1 HgCdTe array, now nearly fifteen years old, which was surplus from the initial Rockwell experiments on the MBE detector fabrication process. Only two quadrants are functional, and it suffers from significant cosmetic defects and numerous non-functioning pixels (Fig 7 (a)). 


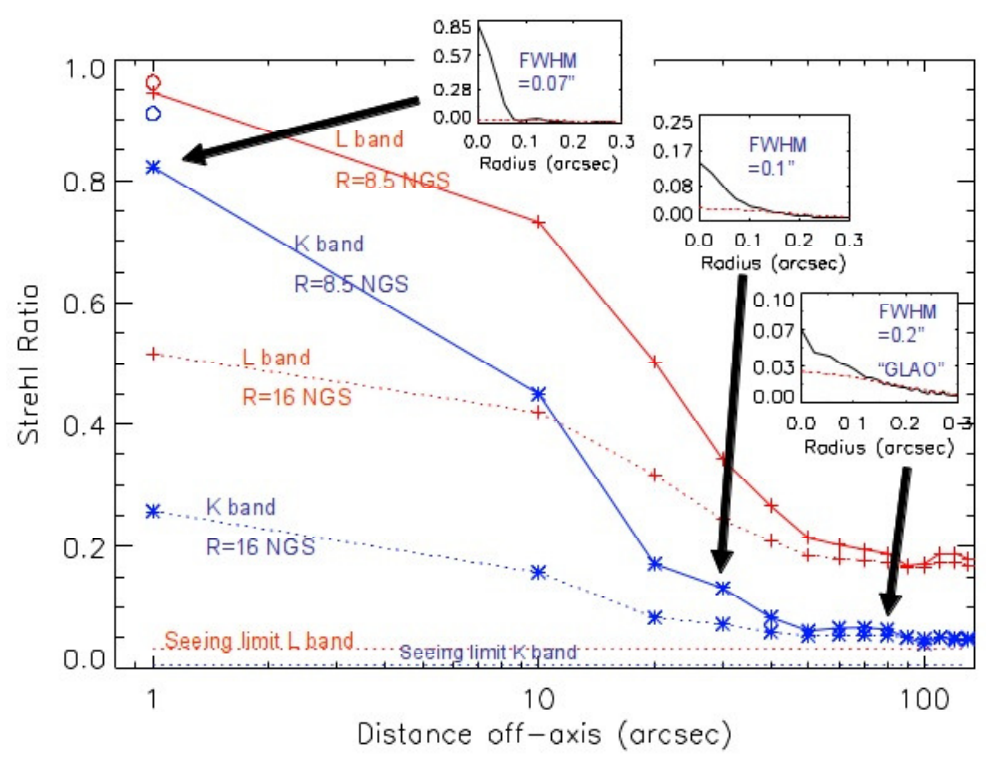

Figure 8. Simulations showing how the wide FOV of the ASM and large patrol field of the PyWFS stages provides "GLAO-like" performance at over 100" in radius from even a 16th mag guide star.

We plan to install a "science grade" 1-5 $\mu$ m detector that is well matched to the high-Strehl, low-background environment provided by MagAO by upgrading the Clio detector to a Teledyne HAWAII-2RG (H2RG) 2048x2048 array. ${ }^{30}$ The detector upgrade will provide numerous improvements, including:

- $5 \mathrm{x}$ reduction in readout noise

- reduction of dark current to well below the lowest backgrounds for Clio

- increased FOV to 33.0" in the coarse plate scale (1200x1200 pixels)

- increased FOV to 21.6" in the fine plate scale (1350x1350 pixels)

- more than an order of magnitude reduction in non-functional pixels

Table 4. Clio Detector Parameters

\begin{tabular}{|l|c|c|}
\hline Parameters & $\begin{array}{c}\text { Clio2 } \\
\text { (current) }\end{array}$ & $\begin{array}{c}\text { Clio3 } \\
\text { (upgrade) }\end{array}$ \\
\hline Detector & HAWAII-1 & Teledyne H2RG \\
Format [pix] & $1024 \times 512$ & $2048 \times 2048$ \\
Coarse FOV ["] & $28 \times 14$ & $33 \times 33^{*}$ \\
Fine Platescale [mas/pix] & 15.9 & 15.9 \\
RON [e- rms] & 100 (measured) & $<21$ (JWST req.) \\
Dark Current [e-/sec/pix] & $\lesssim 20$ (measured) & $<0.1$ (at 55K) \\
\hline
\end{tabular}

The upgraded Clio will take full advantage of the high performance of MagAO-2K. In $\mathrm{J}$ and $\mathrm{H}$ improved noise and cosmetics will dramatically improve sensitivity. This significant improvement in Clio sensitivity and FOV will enable very wide-field off-axis imaging. The PyWFS, by design, can patrol a $\sim 100$ " radius field, giving $>90 \%$ sky-coverage for $R \sim 16$ guide stars at $30^{\circ}$ galactic longitude. ${ }^{31}$ Our simulations (Fig 8 ) show that $>$ 100" off-axis, MagAO provides a "GLAO-like" correction. This effect is modest at $K$ band, and at $L$ provides significant gains over the seeing limit (see the on-sky example in Fig. 7c).

\section{MAGAO-X: A NEW CORONAGRAPHIC EXTREME AO SYSTEM}

We next describe our plans to develop a new visible-to-near-IR "extreme" AO (ExAO) system behind existing MagAO. MagAO-X will be an ExAO system optimized for working in the optical $(\lambda<1 \mu \mathrm{m})$ while providing imaging and spectroscopic capabilities out to $H$ band $(1.6 \mu \mathrm{m})$. It will consist of a 2000 actuator Boston 
Micromachines Corp. (BMC) deformable mirror (DM) controlled by a PyWFS operating at $3.63 \mathrm{kHz}$. This entirely new and self-contained instrument will leverage the existing MagAO system, which provides an initial correction and minimizes the stroke requirement of the new DM. Diffracted starlight will be suppressed using coronagraphs, starting with a vector apodizing phase plate (vAPP) coronagraph, which has been proven on sky. Our final goal is to deploy a cutting-edge, phase-induced amplitude apodization (PIAA) complex mask coronagraph (PIAACMC). ${ }^{32,33}$ This will enable an inner working angle (IWA) of $0.87 \lambda / D$ while maintaining full $D=6.5 \mathrm{~m}$ resolution with high off-axis throughput. With these coronagraphs MagAO-X will employ low-order wavefront sensing (LOWFS) using light rejected either by a focal plane mask or by the Lyot stop. Finally, speckles will be sensed using high-speed, low-noise science detectors as focal plane wavefront sensors (FPWFS).

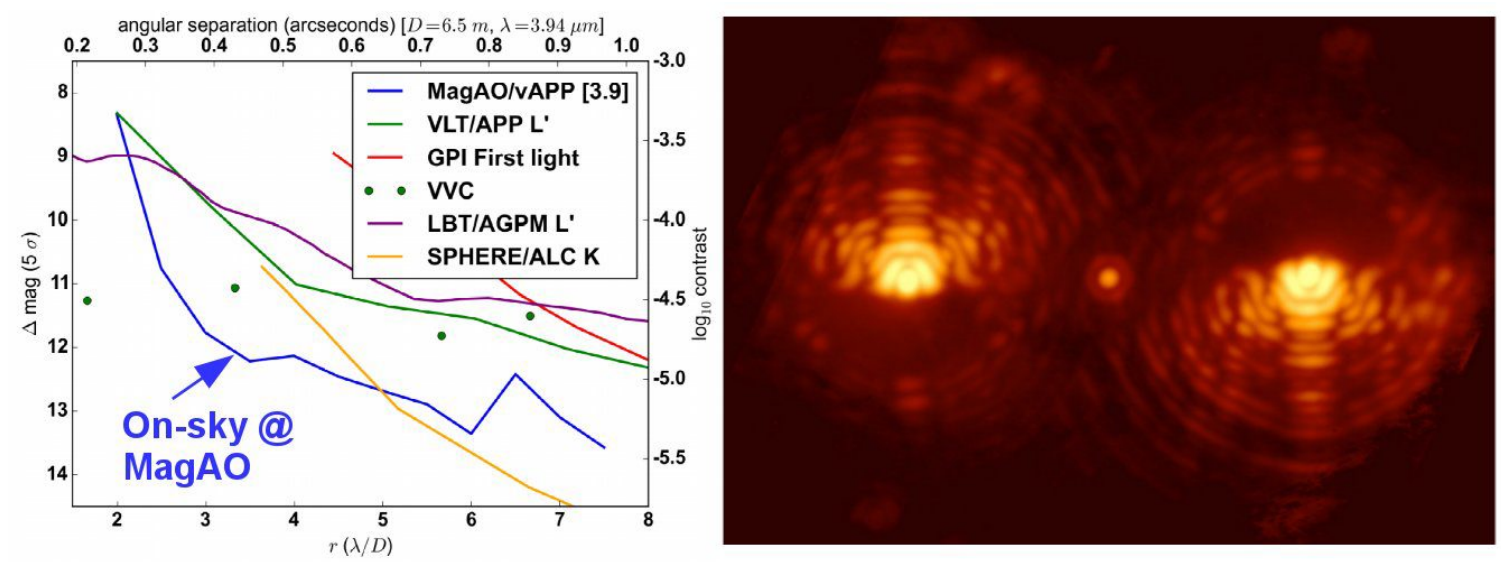

Figure 9. Left: vAPP contrast achieved on-sky in $L$ band with MagAO, compared to other coronagraphs. Right: On-sky vAPP PSF. Note this is one star, split by the device, with dark holes on each side providing $360^{\circ}$ coverage. Also note the central leakage PSF. Figure adapted from Otten et al. ${ }^{34}$

Science focal plane instrumentation initially includes an electron multiplying CCD (EMCCD) for $\lambda \lesssim 1 \mu \mathrm{m}$, with $J-H$ bands $(1.2-1.6 \mu \mathrm{m})$ being sensed by our existing (soon to be upgraded) Clio camera. Later, an energyresolving microwave kinetic inductance detector (MKID) array will provide integral field spectroscopy (IFS) from 0.7-1.4 $\mu \mathrm{m}$. Additional focal plane instruments will be accommodated including the "RHEA@MagAO-X" visible-light $R \sim 60,000$ single-mode fiber IFS.

We plan to adopt a three-phase approach to develop the critical subsystems of MagAO-X, constructing and commissioning manageable subsets of the overall instrument in serial. This approach will allow us to manage the complexity and risk more effectively than possible if the entire instrument were delivered at once. It also more efficiently uses telescope allocations during commissioning, as it provides time to analyze critical on-sky results and re-optimize the instrument between runs. Each of the three phases will be scientifically productive, ensuring that the MagAO-X project will have a major science impact early on while we build to our ultimate scientific goals. Briefly, the three phases are: Phase I: A new PyWFS will drive the existing ASM. A new visible-wavelength vAPP coronagraph, optimized for $H \alpha$, will be introduced. We will exploit the vAPP leakage term (explained in detail below) to feed the LOWFS. Low order aberrations will be fed back to the PyWFS. We will achieve contrasts of $10^{-4}$ from $\sim 2-10 \lambda / D$. Phase II: The new 2000-actuator DM will be brought online, controlling up to 1690 modes at $3630 \mathrm{~Hz}$, to complement the Phase I vAPP and LOWFS. This will realize Strehl ratios of $\sim 80 \%$ at $H \alpha$ on brighter guide stars, and allow high contrast imaging on guide stars as faint as 12 th mag. Phase III: Finally, we will fabricate and deploy PIAACMCs with Lyot-based LOWFS (LLOWFS). We have optimized a high throughput design ( $78 \%$ off-axis) with IWA $=0.87 \lambda / D$, over a broad $20 \%$ bandpass.

\subsection{Phase I - Visible vAPP coronagraph and LOWFS}

In this phase we will introduce a new PyWFS, which will drive the existing ASM. The speed of this combination is limited by the ASM, which (following the MagAO-2K upgrade just described) will be 1800-2000 Hz. A main goal of this phase is to develop interfaces between the new MagAO-X system and the ASM for use as a 
woofer in the following phases. Phase I of MagAO-X will also introduce a vAPP coronagraph combined with a post-coronagraph LOWFS.

PyWFS: The MagAO-X PyWFS detector is a First Light Imaging (FLI) OCAM-2K EMCCD, which has a maximum stable frame rate (demonstrated on sky at SCExAO) of $3.63 \mathrm{kHz}$ in a 120x120 pixel region. This allows for sampling each of the four PyWFS pupils with up to 56x56 pixels to ensure that all controllable modes are sampled with low aliasing. The optical design is almost identical to our very successful MagAO PyWFS. Of crucial importance, we have a precision polished $<5 \mu \mathrm{m}$ tip double-pyramid prism already in hand. The PyWFS has an FSM to support modulation (moving the beam in $3.63 \mathrm{kHz}$ circle about the tip), which increases the linear range. We will also utilize 0 modulation, which reduces sensitivity to photon noise at the cost of lower dynamic range.

vAPP Coronagraph: The APP coronagraph ${ }^{35,36}$ is a pupil-plane only coronagraph which redistributes the diffraction structure of the PSF, resulting in a D-shaped dark hole. The vector-APP (vAPP) exploits the (achromatic) geometric phase imposed by patterned liquid crystals in combination with circular polarization splitting to create dark holes on each side of the $\operatorname{star}^{37}$ providing nearly $360^{\circ}$ "dark-hole" coverage. There is also a small (few \%) leakage term between the two images, which is an otherwise unaltered PSF. The vAPP was demonstrated on-sky in MagAO's Clio camera (see Fig 9 and Otten et al, submitted ${ }^{34}$ ), with $5 \sigma$ contrast $10^{-5}$ at $3 \lambda / D$ after angular differential imaging (ADI) processing. The vAPP has also been demonstrated at visible wavelengths. ${ }^{38}$

VAPP-LOWFS: Using an out of focus image of the leak PSF we will sense the lowest order $(\lesssim 30)$ aberrations. These low order modes will be fed back to the PyWFS as null offsets (a well established capability). Coronagraphic-LOWFS has been demonstrated in the lab ${ }^{39}$ and on-sky in the Subaru Coronagraphic Extreme AO (SCExAO) system $^{40}$ (see also Jovanovic, et al, theses proceedings ${ }^{41}$ ).

SDI: We will utilize the well-proven SDI technique and "ghost-free" filters. Instead of a Wollaston beam splitter (BS), which has significant polarization artifacts and has a $50 \%$ throughput loss, we will use a dichroic-cube BS. In addition to $\mathrm{H} \alpha$ and $\mathrm{H} \beta$, we will offer $[O I]$ and $[S I I]$ SDI as in current VisAO and non-SDI broad g', r', i', z' and $\mathrm{y}$.

\subsection{Phase II - Visible extreme-AO}

In Phase II we reuse many of the optics from Phase I, with the main improvement being the addition of a deformable mirror. The PyWFS will now drive this DM, using the ASM as a woofer to manage stroke. The same vAPP coronagraph and LOWFS combination will be used. Focal plane instruments in Phase II include the Andor 888 EMCCD (with SDI as described above), the Clio IR imager, and the RHEA@MagAO-X IFS.

DM: Wavefront correction is made by a 2000-actuator BMC micro-electro-mechanical systems (MEMS) "2KDM", with $1.5 \mu \mathrm{m}$ stroke and protected silver coating for maximum throughput. It will have $100 \%$ yield (no bad actuators) within our $48.5 \times 49$ actuator (ellipse) illuminated pupil including central obscuration and spiders. Our team has considerable experience working with MEMS DMs. ${ }^{42-45}$ Based on lessons learned at SCExAO, ${ }^{46}$ the DM will be operated under partial vacuum to achieve a $10 \mathrm{kHz}$ response time.

RHEA IFS: With Phase II online, A RHEA-based IFS will be used at MagAO-X (RHEA@MagAO-X, PI: Michael Ireland). Similar to RHEA@Subaru, this is closely based on the Replicable High-resolution Exoplanet and Asteroseismology (RHEA) spectrograph as described in Feger et al. ${ }^{47,48}$ This 9 element IFS will be used for spatially resolved $R \sim 60,000$ spectroscopic observations.

\subsection{Phase III - Visible PIAACMC and LOWFS}

In the final phase we will re-use all the opto-mechanics of Phase II but add a very high performance PIAACMC. The full mechanical design is shown in Fig. 10.

PIAACMC: We will use transmissive $\mathrm{CaF}_{2}$ PIAA lenses for simplicity, ${ }^{49}$ separated by $200 \mathrm{~mm}$ and circularly symmetric for ease of manufacturing. The telescope has a $29 \%$ central obscuration and four secondary supports. A crack in the ASM shell was stop-drilled resulting in a "slot" in the pupil at $\sim 9$ o'clock (it has no detectable impact on MagAO performance). These features were considered in numerically optimizing the complex focal 


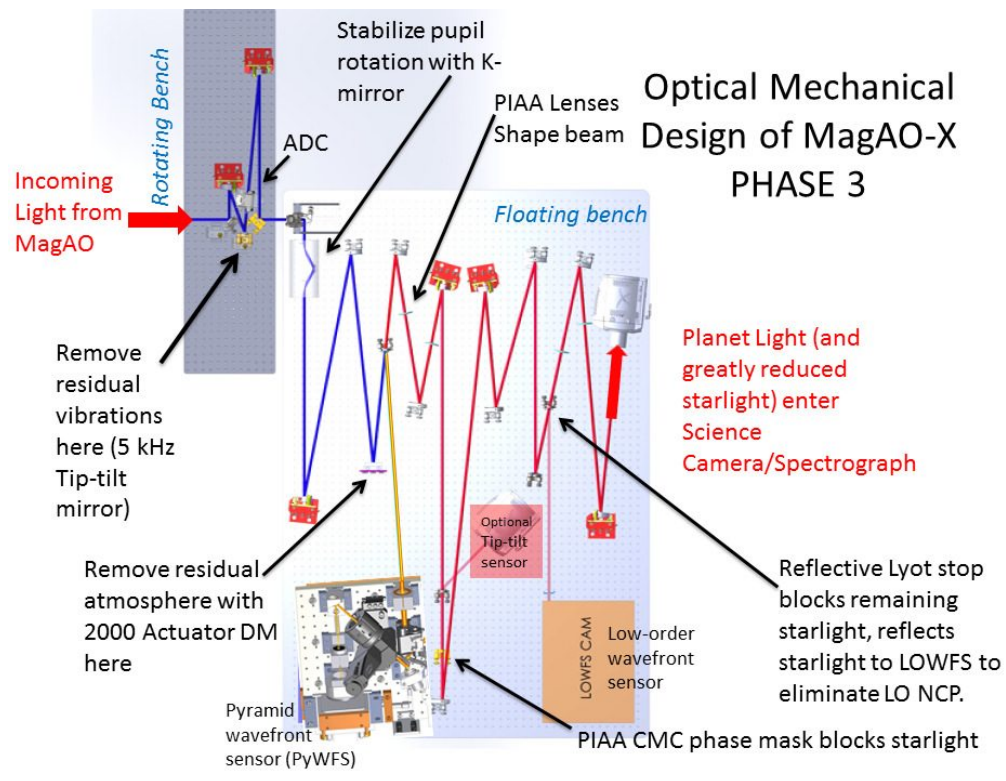

Figure 10. SOLIDWORKS mechanical design detail, top-down view

plane mask (FPM) ${ }^{50}$ This multi-zone $\mathrm{SiO}_{2}$ mask is compatible with current etch manufacturing processes. Masks were designed for $0.656 \mu \mathrm{m}$ and $0.9 \mu \mathrm{m}$ for $20 \%$ wide spectral bands, with outer radius $0.96 \lambda / D(47.5 \mu \mathrm{m}$ in $\mathrm{f} / 57$ at $\lambda=0.9 \mu \mathrm{m})$ at the central wavelength. The sag of each sector was optimized to achieve high contrast across the full spectral band and tolerate moderate stellar angular size. The throughput of the optimized designs is $78 \%$, with an inner working angle of $0.87 \lambda / D$ (defined as the point where throughput is $0.5 \times 0.78$ ). Nearly all star light is redistributed to the central obscuration in the Lyot plane, where it is reflected to the LOWFS. The design is summarized in Fig. 11.

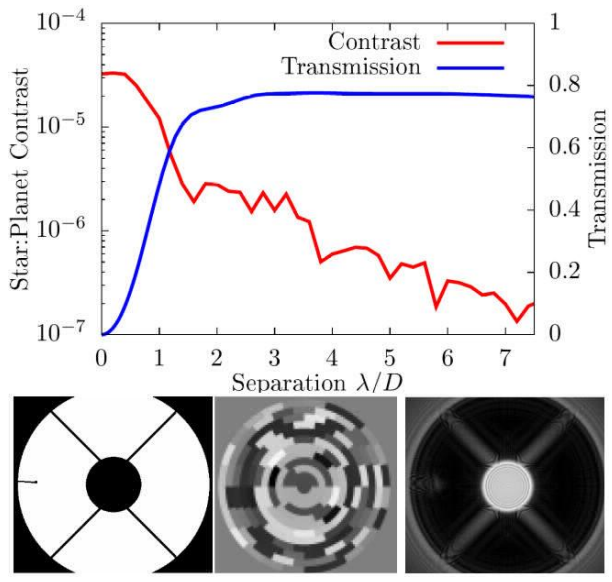

Figure 11. PIAACMC design. Top: contrast and off-axis throughput in 20\% bandpass. Bottom: Left: MagAO-X pupil. Mid: optimized $\mathrm{SiO}_{2}$ mask. Right: Nearly all star light is rejected by the Lyot stop and sent to the LOWFS.

DARKNESS IFS: In Phase III we will bring the DARKNESS IFS (PI: Ben Mazin) to MagAO-X. DARKNESS is based on MKIDs, ${ }^{51,52}$ which can record the energy and arrival time of optical and near-IR photons without read noise or dark current, and have been proven at the Palomar Hale telescope with the ARCONS instrument. ${ }^{53}$ DARKNESS has the unique ability to serve simultaneously as the 0.7-1.4 $\mu \mathrm{m} R \sim 8$ IFS for science data and as a FPWFS. ${ }^{54}$ The high time-resolution of MKIDs with 0 read noise allows speckle control at rates high enough to eliminate atmospheric speckles, which will dramatically improve the achievable contrast ratio of the final MagAO-X system. 


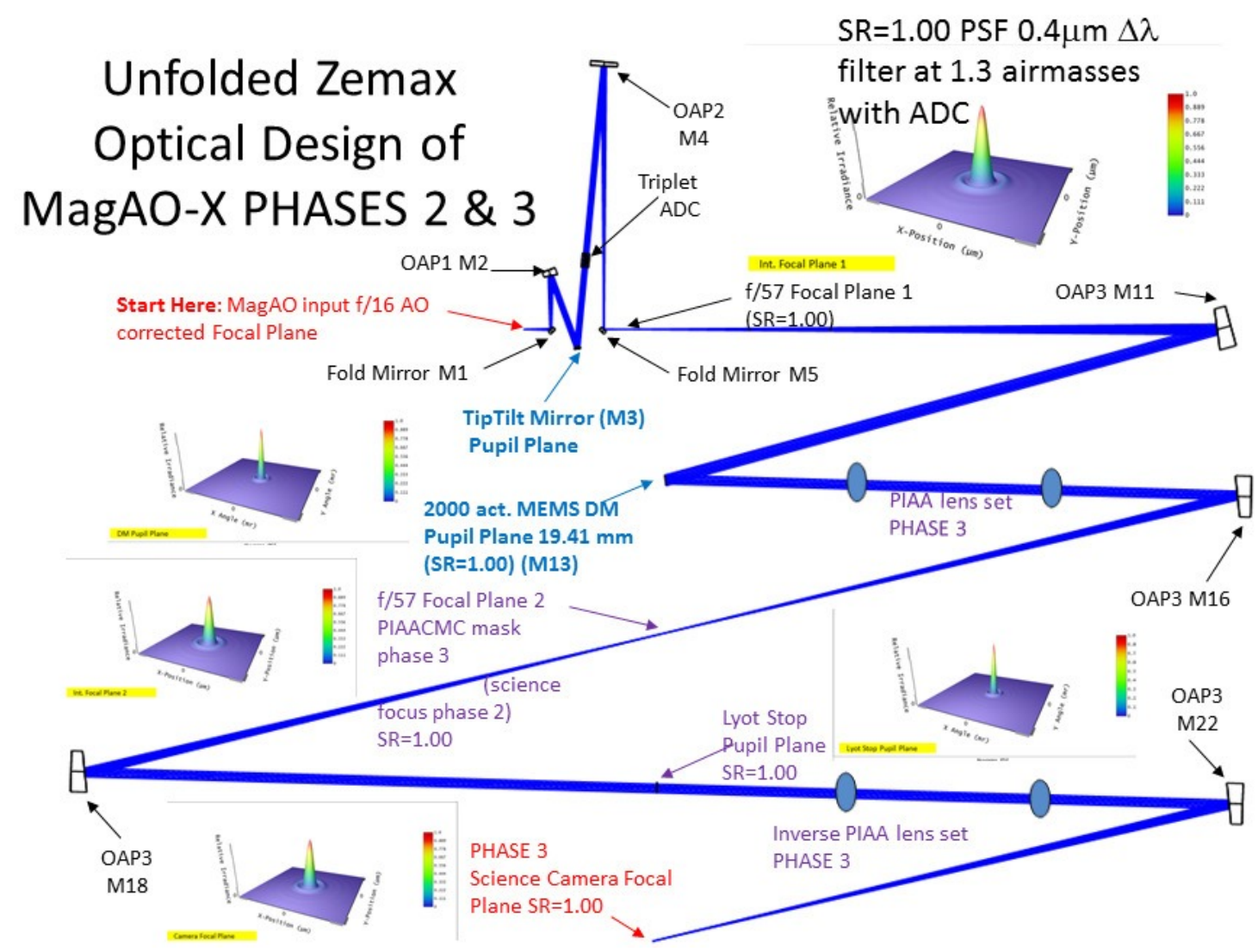

Figure 12. Zemax design Strehl ratio (SR) is 100\%. With the expected polishing errors and alignment errors (see Table 1) the Strehl falls to $99.3 \%$ at $0.9 \mu m$ hence any further loss of Strehl will be from the atmosphere not the MagAO-X optics.

Optical Quality: We have selected off-axis parabolas (OAPs) which produce $\sim 100 \%$ Strehl ratio (SR) PSFs on-axis (Fig. 12) and equally good pupil images at 1.3 airmass over $0.4 \mu \mathrm{m}$ bandpasses with our triplet ADC design (modified from our on-sky proven $\mathrm{ADC}^{55}$ ). These Zemax-calculated SRs assume no atmospheric wavefront errors, and normal ( $\mathrm{T}=12 \mathrm{C}, 0.8$ barr) atmospheric dispersion across the $0.4 \mu \mathrm{m}$ bandpass. The chromatic pupil shear from the ADC is a very acceptable $<0.1 \%$ of the pupil or $<5 \%$ of an actuator over the whole bandpass of the PyWFS $(0.5-1.0 \mu \mathrm{m})$. Table 5 summaries our OAP optics and fabrication tolerances. The design carefully considered the Talbot effect to minimize amplitude errors, though we have deferred an end-to-end Fresnel propagation to preliminary design review (PDR).

\subsection{Performance Analysis}

We have conducted extensive end-to-end closed-loop modeling of MagAO-X using a custom c++ code. The simulated pupil included the central obscuration, spiders, and slot (discussed above). Multi-layer turbulence was simulated using a profile based on the GMT site survey ${ }^{10}$ and LCO statistics. ${ }^{57}$ We used the "median" and "25\%-ile" models described in Section 2.1 above. Fresnel propagation between layers was included (scintillation). We used a 41x41 square Fourier basis augmented with the first 10 Zernikes, and then orthogonalized on the pupil. The PyWFS model is diffractive with cross-talk between quadrants. We calculated slopes ${ }^{58}$ and used a leaky integrator control law. Detector read noise and dark current are included for CCDs. Electron multiplication noise and clock-induced-charge are included for the EMCCD. System throughput accounted for all optics, coatings, and detector QEs. Modal gains were optimized ${ }^{59,60}$ for various star magnitudes.

In Fig. 13 we show performance for $3 \lambda / D$ modulation (typical for existing MagAO), and for $0 \lambda / D$, which has significantly lower photon-noise sensitivity as expected. ${ }^{14}$ Similar results obtain for very small non-zero modulation. Our goal for MagAO-X is to use small to no modulation. Our simulation-based error budget is 
Table 5. Summary of all Reflective Powered Optics in the MagAO-X Zemax Design

\begin{tabular}{|c|c|c|c|c|c|}
\hline $\begin{array}{l}\text { Optic } \\
\text { Name }\end{array}$ & $\begin{array}{l}\text { FS (vertex } \\
\text { foc.) }[\mathrm{mm}]\end{array}$ & $\begin{array}{l}\text { FP (parent } \\
\text { foc.) }[\mathrm{mm}]\end{array}$ & $\begin{array}{c}\text { Off Axis } \\
\text { Dist. }[\mathrm{mm}]\end{array}$ & $\begin{array}{l}\text { Effective } \\
\text { focal ratio }\end{array}$ & $\begin{array}{l}\text { Purpose and performance of the }(2 \\
\text { inch diameter) optic }\end{array}$ \\
\hline OAP 1 (M2) & 150.00 & 145.5872 & 50.69 & 16.16 & $\begin{array}{l}\text { Forms }>99 \% \text { SR } 9.282 \mathrm{~mm} \text { dia. pupil } \\
\text { in collimated space on } \mathrm{T} / \mathrm{T} \text { mirror. }\end{array}$ \\
\hline OAP2 (M4) & 529.03 & 527.8459 & 50.00 & 56.995 & $\begin{array}{l}\text { M4 takes pupil at infinity and forms } \\
>99 \% \text { SR f/57 focus (single) }\end{array}$ \\
\hline $\begin{array}{l}\text { OAP3 } \\
\text { (M11, M16) } \\
(\mathrm{M} 18, \mathrm{M} 22)\end{array}$ & 1106.27 & 1087.422 & 286.32 & 56.995 & $\begin{array}{l}\text { M11/M16 relay the }>99 \% \text { SR } \\
19.62 \times 19.40 \mathrm{~mm} \text { pupil onto the } \\
49 \times 48.5 \text { act. DM, then form }>99 \% \\
\text { SR f/57 focus on the PIAA FPM. } \\
\text { M18/M22 relay pupil to Lyot stop, } \\
\text { form final }>99 \% \text { Strehl } / 57 \text { focus. }\end{array}$ \\
\hline OAP 0 & 313.66 & 311.5441 & 51.23 & 16.15 & $\begin{array}{l}\text { Pair produces telescope pupil and } \\
>99 \% \text { SR f } / 16.16 \text { focus (lab tests only) }\end{array}$ \\
\hline \multicolumn{6}{|c|}{$\begin{array}{l}\text { Quotes received from Aperture Optical Sciences Inc. (AOS), CT, and Space Optics Research Labs } \\
\text { (SORL), MT, for } \lambda / 20 \mathrm{rms} \text { overall surface quality. Tolerance: low spatial frequency }(s>5 \mathrm{~mm} \text { at DM) } \\
\text { surface errors }<16 \mathrm{~nm} \mathrm{rms} \text {; mid spatial freq. }(0.1<s<5 \mathrm{~mm}):<3.2 \mathrm{~nm} \mathrm{rms} \text {;igh spatial freq. ( }<0.1 \\
\mathrm{~mm}):<2.6 \mathrm{~nm} \text { rms. Such specifications have been demonstrated }(12 \pm 3 \mathrm{~nm} \mathrm{rms}) \text { by the HiCAT testbed } \\
\text { without DM correction }{ }^{56} \text { and are well within our MagAO-X error budget. }\end{array}$} \\
\hline
\end{tabular}

Table 6. Error Budget for 5th mag star

\begin{tabular}{|c|c|c|c|}
\hline Term & \multicolumn{3}{|c|}{ WFE [nm rms] } \\
\hline \multicolumn{4}{|c|}{ Dynamic Atmospheric Errors } \\
\hline Measurement & \multicolumn{3}{|c|}{4.7} \\
\hline Servo Lag & \multicolumn{3}{|c|}{28.5} \\
\hline Fitting & \multicolumn{3}{|c|}{35.4} \\
\hline Aliasing & \multicolumn{3}{|c|}{3} \\
\hline Sub-ap shear & \multicolumn{3}{|c|}{2} \\
\hline \multicolumn{4}{|c|}{ Total Dynamic WFE: $45.8 \mathrm{~nm} \mathrm{rms}$} \\
\hline \multicolumn{4}{|c|}{ Static NCP and Unsensed } \\
\hline & $\mathrm{LO}$ & $\mathrm{MO}$ & $\mathrm{HO}$ \\
\hline OAPs X 3 & 16 & 3.2 & 2.6 \\
\hline Flats X 7 & 16 & 3.2 & 2.6 \\
\hline DM: Surface & 26 & \multicolumn{2}{|c|}{15} \\
\hline DM: Flat & 35 & \multicolumn{2}{|c|}{20} \\
\hline Alignment & $<5$ & \multicolumn{2}{|c|}{$<2$} \\
\hline Flexure & $<5$ & \multicolumn{2}{|c|}{$<2$} \\
\hline \multicolumn{4}{|c|}{ post-LOWFS Static WFE: $28 \mathrm{~nm}$ rms } \\
\hline \multicolumn{4}{|c|}{ Total WFE: $53.7 \mathrm{~nm} \mathrm{rms}$} \\
\hline \multicolumn{4}{|c|}{$H \alpha$ Strehl Ratio: $\mathbf{7 7 \%}$} \\
\hline
\end{tabular}

shown in Table 6. The non-common-path (NCP) and unsensed errors were estimated based on the above optical design. These errors are included in Fig. 13. In all phases the LOWFS will remove NCP errors with spatial frequency $k \lesssim 4 / D$ to well below the residual atmosphere. Outside $4 \lambda / D$ the FPWFS will sense and remove these, mitigating their impact in Phase III. Additional speckle control is provided in post-processing. ${ }^{61,62}$ In Fig. 14 we show the resulting PSFs with and without the coronagraph, and contrast predictions.

\section{CONCLUSION}

We have described upgrades to the MagAO system, both in progress and planned for the near future. The NSF-funded MagAO-2K project, which is in progress, will 


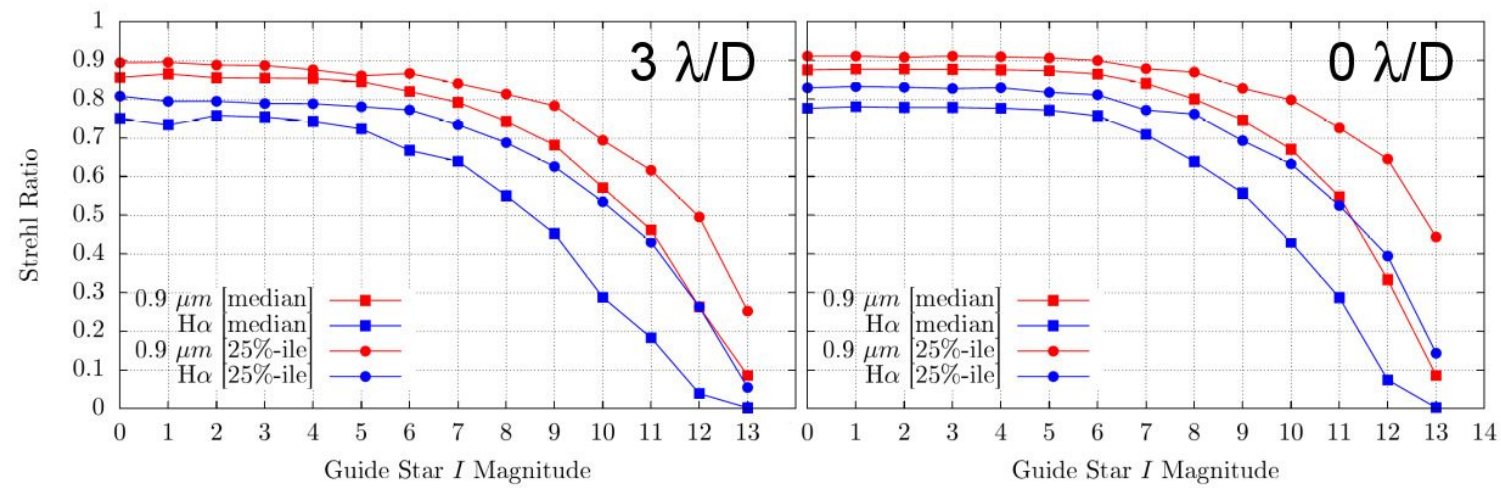

Figure 13. Strehl ratio vs. guide star magnitude from closed-loop simulations. For $3 \lambda / D$ modulation (left) and $0 \lambda / D$ (right).
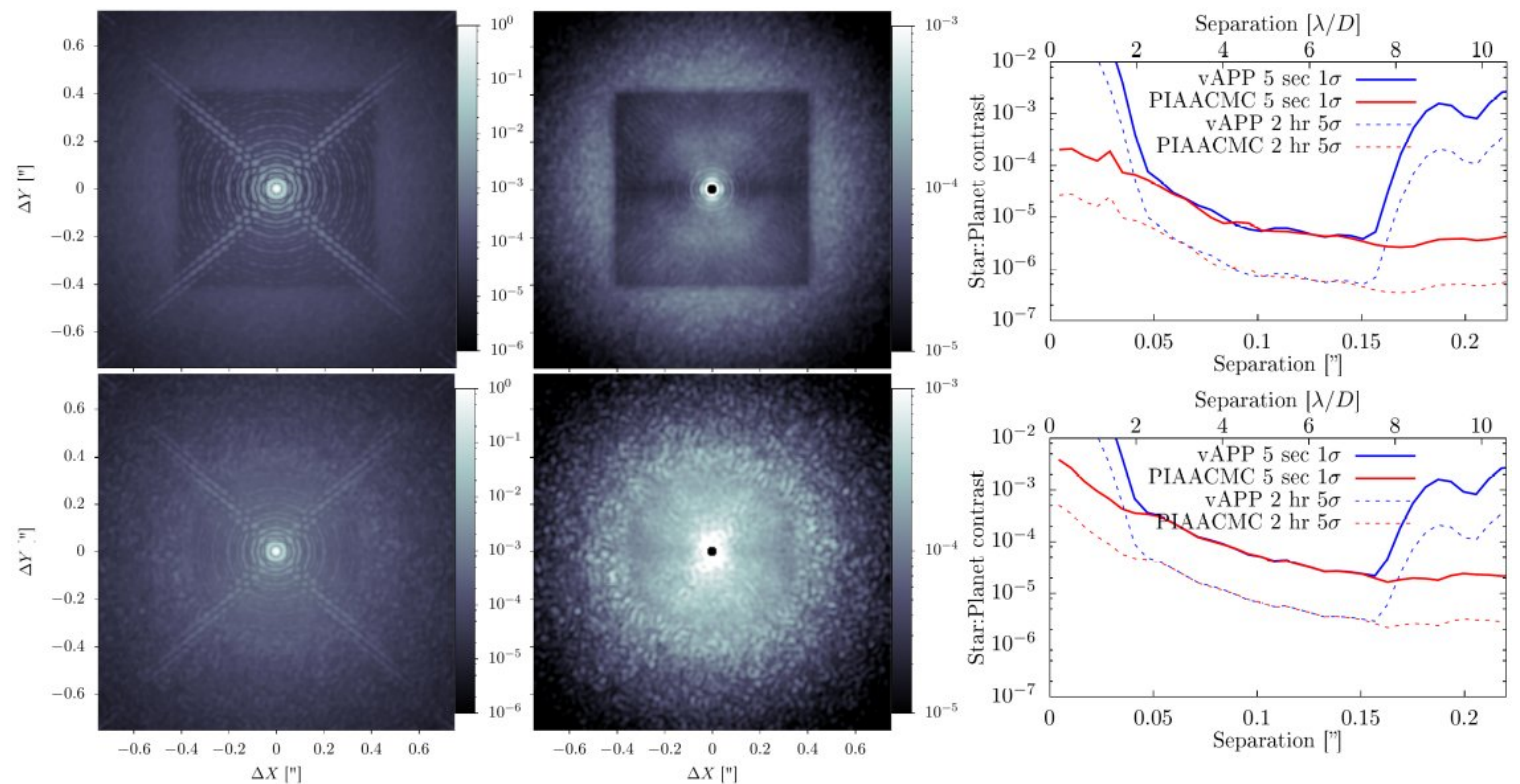

Figure 14. End-to-end closed-loop simulations at $H \alpha$. Top row: 5th mag star, median conditions. Bottom row: 10th mag star, 25\%-ile. Left: Simulated PSF w/out coronagraph. Middle: post-coronagraph, inner $0.87 \lambda / D$ masked. Right: contrast. 
- Re-calibrate the MagAO adaptive secondary mirror (ASM) position control system

- Increase PyWFS sampling to 30x30

- Improve ASM update rate to $1500-2000 \mathrm{~Hz}$

- Implement advanced vibration control algorithms

- Upgrade the Clio IR camera to an H2RG detector.

MagAO-X will build upon the success of MagAO and the improvements of MagAO-2K by developing a visible coronagraphic ExAO capability. This new AO system will include

- A BMC 2000 actuator MEMS DM

- An OCAM-2K based PyWFS running at $3.63 \mathrm{kHz}$;

$\bullet \sim 1700$ controlled modes

- Cutting-edge coronagraphs, such as the vAPP and PIAACMC.

- Coronagraphic LOWFS

- A suite of focal plane instruments including imagers and spectrographs enabling high-contrast and highresolution science.

These improvements will ensure that MagAO continues to take full advantage of its excellent Las Campanas Observatory site, and help lay the groundwork for ExAO on the GMT.

\section{ACKNOWLEDGMENTS}

J.R.M was supported under contract with the California Institute of Technology (Caltech) funded by NASA through the Sagan Fellowship Program. KMM's and LMC's work is supported by the NASA Exoplanets Research Program (XRP) by cooperative agreement NNX16AD44G. This material is based upon work supported by the National Science Foundation under Grant No. 1506818. This paper includes data gathered with the 6.5 meter Magellan Telescopes located at Las Campanas Observatory, Chile. This research has made use of NASA's Astrophysics Data System Bibliographic Services.

\section{REFERENCES}

[1] Macintosh, B., Graham, J. R., Barman, T., De Rosa, R. J., Konopacky, Q., Marley, M. S., Marois, C., Nielsen, E. L., Pueyo, L., Rajan, A., Rameau, J., Saumon, D., Wang, J. J., Patience, J., Ammons, M., Arriaga, P., Artigau, E., Beckwith, S., Brewster, J., Bruzzone, S., Bulger, J., Burningham, B., Burrows, A. S., Chen, C., Chiang, E., Chilcote, J. K., Dawson, R. I., Dong, R., Doyon, R., Draper, Z. H., Duchêne, G., Esposito, T. M., Fabrycky, D., Fitzgerald, M. P., Follette, K. B., Fortney, J. J., Gerard, B., Goodsell, S., Greenbaum, A. Z., Hibon, P., Hinkley, S., Cotten, T. H., Hung, L.-W., Ingraham, P., Johnson-Groh, M., Kalas, P., Lafreniere, D., Larkin, J. E., Lee, J., Line, M., Long, D., Maire, J., Marchis, F., Matthews, B. C., Max, C. E., Metchev, S., Millar-Blanchaer, M. A., Mittal, T., Morley, C. V., Morzinski, K. M., Murray-Clay, R., Oppenheimer, R., Palmer, D. W., Patel, R., Perrin, M. D., Poyneer, L. A., Rafikov, R. R., Rantakyrö, F. T., Rice, E. L., Rojo, P., Rudy, A. R., Ruffio, J.-B., Ruiz, M. T., Sadakuni, N., Saddlemyer, L., Salama, M., Savransky, D., Schneider, A. C., Sivaramakrishnan, A., Song, I., Soummer, R., Thomas, S., Vasisht, G., Wallace, J. K., Ward-Duong, K., Wiktorowicz, S. J., Wolff, S. G., and Zuckerman, B., "Discovery and spectroscopy of the young jovian planet 51 Eri b with the Gemini Planet Imager," Science 350, 64-67 (2015).

[2] Sallum, S., Follette, K. B., Eisner, J. A., Close, L. M., Hinz, P., Kratter, K., Males, J., Skemer, A., Macintosh, B., Tuthill, P., Bailey, V., Defrère, D., Morzinski, K., Rodigas, T., Spalding, E., Vaz, A., and Weinberger, A. J., "Accreting protoplanets in the LkCa 15 transition disk," Nature 527, 342-344 (2015). 
[3] Wagner, K., Apai, D., Kasper, M., Kratter, K., McClure, M., Robberto, M., and Beuzit, J.-L., "Direct Imaging Discovery of a Jovian Exoplanet Within a Triple Star System," ArXiv e-prints (2016).

[4] Guyon, O., Martinache, F., Cady, E. J., et al., "How ELTs will acquire the first spectra of rocky habitable planets," Proc. SPIE 8447, 84471X (2012).

[5] Males, J. R., Close, L. M., Guyon, O., Morzinski, K., Puglisi, A., Hinz, P., Follette, K. B., Monnier, J. D., Tolls, V., Rodigas, T. J., Weinberger, A., Boss, A., Kopon, D., Wu, Y.-l., Esposito, S., Riccardi, A., Xompero, M., Briguglio, R., and Pinna, E., "Direct imaging of exoplanets in the habitable zone with adaptive optics," Proc. SPIE 9148, 914820 (2014).

[6] Close, L. M., Males, J. R., Morzinski, K., Kopon, D., Follette, K., Rodigas, T. J., Hinz, P., Wu, Y.-L., Puglisi, A., Esposito, S., Riccardi, A., Pinna, E., Xompero, M., Briguglio, R., Uomoto, A., and Hare, T., "Diffraction-limited Visible Light Images of Orion Trapezium Cluster with the Magellan Adaptive Secondary Adaptive Optics System (MagAO)," ApJ 774, 94 (2013).

[7] Morzinski, K. M., Close, L. M., Males, J. R., Kopon, D., Hinz, P. M., Esposito, S., Riccardi, A., Puglisi, A., Pinna, E., Briguglio, R., Xompero, M., Quirós-Pacheco, F., Bailey, V., Follette, K. B., Rodigas, T. J., Wu, Y.-L., Arcidiacono, C., Argomedo, J., Busoni, L., Hare, T., Uomoto, A., and Weinberger, A., "MagAO: Status and on-sky performance of the Magellan adaptive optics system," Proc. SPIE 9148, 4 (2014).

[8] Morzinski, K. M., Males, J. R., Skemer, A. J., Close, L. M., Hinz, P. M., Rodigas, T. J., Puglisi, A., Esposito, S., Riccardi, A., Pinna, E., Xompero, M., Briguglio, R., Bailey, V. P., Follette, K. B., Kopon, D., Weinberger, A. J., and Wu, Y.-L., "Magellan Adaptive Optics First-light Observations of the Exoplanet $\beta$ Pic b. II. 3-5 $\mu \mathrm{m}$ Direct Imaging with MagAO+Clio, and the Empirical Bolometric Luminosity of a Self-luminous Giant Planet," ApJ 815, 108 (2015).

[9] Males, J. R., Close, L. M., Morzinski, K. M., Wahhaj, Z., Liu, M. C., Skemer, A. J., Kopon, D., Follette, K. B., Puglisi, A., Esposito, S., Riccardi, A., Pinna, E., Xompero, M., Briguglio, R., Biller, B. A., Nielsen, E. L., Hinz, P. M., Rodigas, T. J., Hayward, T. L., Chun, M., Ftaclas, C., Toomey, D. W., and Wu, Y.-L., "Magellan Adaptive Optics First-light Observations of the Exoplanet $\beta$ Pic B. I. Direct Imaging in the Far-red Optical with MagAO+VisAO and in the Near-ir with NICI," ApJ 786, 32 (2014).

[10] Prieto, G., Thomas-Osip, J. E., Phillips, M. M., McCarthy, P., and Johns, M., "Giant Magellan Telescope site testing seeing and turbulence statistics," Proc. SPIE 7733, 773340 (2010).

[11] Floyd, D. J. E., Thomas-Osip, J., and Prieto, G., "Seeing, Wind, and Outer Scale Effects on Image Quality at the Magellan Telescopes," PASP 122, 731-742 (2010).

[12] Hardy, J. W., [Adaptive Optics for Astronomical Telescopes] (1998).

[13] Males, J. R., Close, L. M., Kopon, D., Quiros-Pacheco, F., Riccardi, A., Xompero, M., Puglisi, A., Gasho, V., Morzinski, K. M., and Follette, K. B., "Laboratory demonstration of real time frame selection with Magellan AO," Proc. SPIE 8447, 844742 (2012).

[14] Guyon, O., "Limits of Adaptive Optics for High-Contrast Imaging," ApJ 629, 592-614 (2005).

[15] Bohlin, R. C., "HST Stellar Standards with 1\% Accuracy in Absolute Flux," in [The Future of Photometric, Spectrophotometric and Polarimetric Standardization], Sterken, C., ed., Astronomical Society of the Pacific Conference Series 364, 315 (2007).

[16] Carbillet, M., Verinaud, C., Guarracino, M., Fini, L., Lardiere, O., Le Roux, B., Puglisi, A. T., Femenia, B., Riccardi, A., Anconelli, B., Correia, S., Bertero, M., and Boccacci, P., "CAOS: a numerical simulation tool for astronomical adaptive optics (and beyond)," Proc. SPIE 5490, 637-648 (2004).

[17] Males, J. R., Close, L. M., Kopon, D., Gasho, V., and Follette, K., "Frame selection techniques for the Magellan adaptive optics VisAO camera," Proc. SPIE 7736, 773660 (2010).

[18] Esposito, S., Riccardi, A., Quirós-Pacheco, F., Pinna, E., Puglisi, A., Xompero, M., Briguglio, R., Busoni, L., Fini, L., Stefanini, P., Brusa, G., Tozzi, A., Ranfagni, P., Pieralli, F., Guerra, J. C., Arcidiacono, C., and Salinari, P., "Laboratory characterization and performance of the high-order adaptive optics system for the Large Binocular Telescope," Ap. Opt. 49, G174 (2010).

[19] Hartung, M., Hayward, T., Saddlemyer, L., Poyneer, L., Cardwell, A., Cavedoni, C., Cho, M., Chilcote, J. K., Collins, P., Dillon, D., Galvez, R., Gausachs, G., Goodsell, S., Guesalaga, A., Hibon, P., Larkin, J., Macintosh, B., Palmer, D., Sadakuni, N., Savransky, D., Serio, A., Rantakyr, F., and Wallace, K., "On-sky vibration environment for the gemini planet imager and mitigation effort," Proc. SPIE 9148, 91480N-91480N-12 (2014). 
[20] Garcés, J., Fernández, S. Z., Close, L. M., Males, J. R., Morzinski, K. M., Escárate, P., R., M. A. C., Clavería, J. L. M., and Zagals, D. A. R., "Vibrations in MagAO: resonance sources identification and first approaches for modeling and control," in [Adaptive Optics Systems V], Proc. SPIE 9909, 9909-141 (2016).

[21] Fernández, S. Z., Close, J. G. L. M., Males, J. R., Morzinski, K. M., Escárate, P., R., M. A. C., Clavería, J. L. M., and Zagals, D. A. R., "Vibrations in MagAO: frequency-based analysis of on-sky data, resonance sources identification, and future challenges in vibrations mitigation," in [Adaptive Optics Systems V], Proc. SPIE 9909, 9909-153 (2016).

[22] Petit, C., Sauvage, J.-F., Fusco, T., Sevin, A., Suarez, M., Costille, A., Vigan, A., Soenke, C., Perret, D., Rochat, S., Barrufolo, A., Salasnich, B., Beuzit, J.-L., Dohlen, K., Mouillet, D., Puget, P., Wildi, F., Kasper, M., Conan, J.-M., Kulcsr, C., and Raynaud, H.-F., "Sphere extreme ao control scheme: final performance assessment and on sky validation of the first auto-tuned lqg based operational system," Proc. SPIE 9148, 91480O-91480O-17 (2014).

[23] Poyneer, L. A., De Rosa, R. J., Macintosh, B., Palmer, D. W., Perrin, M. D., Sadakuni, N., Savransky, D., Bauman, B., Cardwell, A., Chilcote, J. K., Dillon, D., Gavel, D., Goodsell, S. J., Hartung, M., Hibon, P., Rantakyrö, F. T., Thomas, S., and Veran, J.-P., "On-sky performance during verification and commissioning of the Gemini Planet Imager's adaptive optics system," Proc. SPIE 9148, 9148 (2014).

[24] Agapito, G., Quiros-Pacheco, F., Tesi, P., Esposito, S., and Xompero, M., "Optimal control techniques for the adaptive optics system of the LBT," Proc. SPIE 7015, 70153G (2008).

[25] Biller, B. A., Males, J., Rodigas, T., Morzinski, K., Close, L. M., Juhász, A., Follette, K. B., Lacour, S., Benisty, M., Sicilia-Aguilar, A., Hinz, P. M., Weinberger, A., Henning, T., Pott, J.-U., Bonnefoy, M., and Köhler, R., "An Enigmatic Point-like Feature within the HD 169142 Transitional Disk," ApJL 792, L22 (2014).

[26] Stone, J. M., Eisner, J., Skemer, A., Morzinski, K. M., Close, L., Males, J., Rodigas, T. J., Hinz, P., and Puglisi, A., "L-BAND SPECTROSCOPY WITH MAGELLAN-AO/CLIO2: FIRST RESULTS ON YOUNG LOW-MASS COMPANIONS," submitted to ApJ (2016).

[27] Skemer, A. J., Marley, M. S., Hinz, P. M., Morzinski, K. M., Skrutskie, M. F., Leisenring, J. M., Close, L. M., Saumon, D., Bailey, V. P., Briguglio, R., Defrere, D., Esposito, S., Follette, K. B., Hill, J. M., Males, J. R., Puglisi, A., Rodigas, T. J., and Xompero, M., "Directly Imaged L-T Transition Exoplanets in the Mid-infrared," ApJ 792, 17 (2014).

[28] Freed, M., Hinz, P. M., Meyer, M. R., Milton, N. M., and Lloyd-Hart, M., "Clio: a 5- $\mu$ m camera for the detection of giant exoplanets," Proc. SPIE 5492, 1561-1571 (2004).

[29] Hinz, P. M., Heinze, A. N., Sivanandam, S., Miller, D. L., Kenworthy, M. A., Brusa, G., Freed, M., and Angel, J. R. P., "Thermal Infrared Constraint to a Planetary Companion of Vega with the MMT Adaptive Optics System," ApJ 653, 1486-1492 (2006).

[30] Loose, M., Farris, M. C., Garnett, J. D., Hall, D. N. B., and Kozlowski, L. J., "HAWAII-2RG: a $2 \mathrm{k}$ x 2k CMOS multiplexer for low and high background astronomy applications," Proc. SPIE 4850, 867-879 (2003).

[31] Roddier, F., [Adaptive Optics in Astronomy] (2004).

[32] Guyon, O., "Phase-induced amplitude apodization of telescope pupils for extrasolar terrestrial planet imaging," AAP 404, 379-387 (2003).

[33] Guyon, O., Martinache, F., Belikov, R., and Soummer, R., "High Performance PIAA Coronagraphy with Complex Amplitude Focal Plane Masks," ApJS 190, 220-232 (2010).

[34] Otten, G. P. P. L., Snik, F., Kenworthy, M. A., Keller, C. U., Males, J. R., Morzinski, K. M., Close, L. M., Codona, J. L., Montoya, M., Hinz, P. M., Hornburg, K. J., Brickson, L. L., Komanduri, R. K., and Escuti, M. J., "On-sky performance analysis of the vector Apodizing Phase Plate coronagraph on MagAO/Clio2," submitted to ApJ (2016).

[35] Codona, J. L., Kenworthy, M. A., Hinz, P. M., Angel, J. R. P., and Woolf, N. J., "A high-contrast coronagraph for the MMT using phase apodization: design and observations at 5 microns and $2 \lambda / \mathrm{D}$ radius," Proc. SPIE 6269, 62691N (2006).

[36] Kenworthy, M. A., Codona, J. L., Hinz, P. M., Angel, J. R. P., Heinze, A., and Sivanandam, S., "First On-Sky High-Contrast Imaging with an Apodizing Phase Plate," ApJ 660, 762-769 (2007). 
[37] Snik, F., Otten, G., Kenworthy, M., Miskiewicz, M., Escuti, M., Packham, C., and Codona, J., "The vectorAPP: a broadband apodizing phase plate that yields complementary PSFs," Proc. SPIE 8450, 84500M (2012).

[38] Otten, G. P. P. L., Snik, F., Kenworthy, M. A., Miskiewicz, M. N., and Escuti, M. J., "Performance characterization of a broadband vector Apodizing Phase Plate coronagraph," Optics Express 22, 30287 (2014).

[39] Guyon, O., Matsuo, T., and Angel, R., "Coronagraphic Low-Order Wave-Front Sensor: Principle and Application to a Phase-Induced Amplitude Coronagraph," ApJ 693, 75-84 (2009).

[40] Singh, G., Lozi, J., Guyon, O., Baudoz, P., Jovanovic, N., Martinache, F., Kudo, T., Serabyn, E., and Kuhn, J., "On-Sky Demonstration of Low-Order Wavefront Sensing and Control with Focal Plane Phase Mask Coronagraphs," PASP 127, 857-869 (2015).

[41] Jovanovic, N., Guyon, O., Lozi, J., Currie, T., Hagelberg, J., Norris, B. R., Singh, G., Pathak, P., Doughty, D., Goebel, S. B., Males, J. R., Kuhn, J. G., Serabyn, E., Tuthill, P. G., Schworer, G., Martinache, F., Kudo, T., Kawahara, H., Kotani, T., Ireland, M. J., Feger, T., Rains, A. D., Bento, J., Schwab, C., Coutts, D. W., Cvetojevic, N., Gross, S., Arriola, A., Lagadec, T., Kasdin, J., Groff, T., Mazin, B., Minowa, Y., Takato, N., and Tamura, M., "The SCExAO high contrast imager: transitioning from commissioning to science," in [Adaptive Optics Systems V], Proc. SPIE 9909, 9909-34 (2016).

[42] Morzinski, K. M., Evans, J. W., Severson, S., Macintosh, B., Dillon, D., Gavel, D., Max, C., and Palmer, D., "Characterizing the potential of MEMS deformable mirrors for astronomical adaptive optics," Proc. SPIE 6272, 627221 (2006).

[43] Morzinski, K., Macintosh, B., Gavel, D., and Dillon, D., "Stroke saturation on a MEMS deformable mirror for woofer-tweeter adaptive optics," Optics Express 17, 5829 (2009).

[44] Morzinski, K., Johnson, L. C., Gavel, D. T., Grigsby, B., Dillon, D., Reinig, M., and Macintosh, B. A., "Performance of MEMS-based visible-light adaptive optics at Lick Observatory: closed- and open-loop control," Proc. SPIE 7736, 773610 (2010).

[45] Morzinski, K. M., Norton, A. P., Evans, J. W., Reza, L., Severson, S. A., Dillon, D., Reinig, M., Gavel, D. T., Cornelissen, S., Macintosh, B. A., and Max, C. E., "MEMS practice: from the lab to the telescope," Proc. SPIE 8253, 825304 (2012).

[46] Jovanovic, N., Martinache, F., Guyon, O., Clergeon, C., Singh, G., Kudo, T., Garrel, V., Newman, K., Doughty, D., Lozi, J., Males, J., Minowa, Y., Hayano, Y., Takato, N., Morino, J., Kuhn, J., Serabyn, E., Norris, B., Tuthill, P., Schworer, G., Stewart, P., Close, L., Huby, E., Perrin, G., Lacour, S., Gauchet, L., Vievard, S., Murakami, N., Oshiyama, F., Baba, N., Matsuo, T., Nishikawa, J., Tamura, M., Lai, O., Marchis, F., Duchene, G., Kotani, T., and Woillez, J., "The Subaru Coronagraphic Extreme Adaptive Optics System: Enabling High-Contrast Imaging on Solar-System Scales," PASP 127, 890-910 (2015).

[47] Feger, T., Bacigalupo, C., Bedding, T. R., Bento, J., Coutts, D. W., Ireland, M. J., Parker, Q. A., Rizzuto, A., and Spaleniak, I., "RHEA: the ultra-compact replicable high-resolution exoplanet and Asteroseismology spectrograph," Proc. SPIE 9147, 91477I (2014).

[48] Feger, T., Ireland, M. J., Schwab, C., Bento, J., Bacigalupo, C., and Coutts, D. W., "Attaining m/s level intrinsic Doppler precision with RHEA, a low-cost single-mode spectrograph," Submitted to Ex. Astron. (2016).

[49] Lozi, J., Martinache, F., and Guyon, O., "Phase-Induced Amplitude Apodization on Centrally Obscured Pupils: Design and First Laboratory Demonstration for the Subaru Telescope Pupil," PASP 121, 1232-1244 (2009).

[50] Guyon, O., Hinz, P. M., Cady, E., Belikov, R., and Martinache, F., "High Performance Lyot and PIAA Coronagraphy for Arbitrarily Shaped Telescope Apertures," ApJ 780, 171 (2014).

[51] Day, P. K., LeDuc, H. G., Mazin, B. A., Vayonakis, A., and Zmuidzinas, J., "A broadband superconducting detector suitable for use in large arrays," Nature 425, 817-821 (2003).

[52] Mazin, B. A., Bumble, B., Meeker, S. R., O'Brien, K., McHugh, S., and Langman, E., "A superconducting focal plane array for ultraviolet, optical, and near-infrared astrophysics," Optics Express 20, 1503 (2012).

[53] Mazin, B. A., Meeker, S. R., Strader, M. J., Szypryt, P., Marsden, D., van Eyken, J. C., Duggan, G. E., Walter, A. B., Ulbricht, G., Johnson, M., Bumble, B., O"Brien, K., and Stoughton, C., "ARCONS: A 2024 Pixel Optical through Near-IR Cryogenic Imaging Spectrophotometer," PASP 125, 1348-1361 (2013). 
[54] Martinache, F., Guyon, O., Jovanovic, N., Clergeon, C., Singh, G., and Kudo, T., "On-sky speckle nulling with the Subaru Coronagraphic Extreme AO (SCExAO) instrument," Proc. of SPIE 9148, 914821 (2014).

[55] Kopon, D., Close, L. M., Males, J. R., and Gasho, V., "Design, Implementation, and On-Sky Performance of an Advanced Apochromatic Triplet Atmospheric Dispersion Corrector for the Magellan Adaptive Optics System and VisAO Camera," PASP 125, 966-975 (2013).

[56] N'Diaye, M., Choquet, E., Egron, S., Pueyo, L., Leboulleux, L., Levecq, O., Perrin, M. D., Elliot, E., Wallace, J. K., Hugot, E., Marcos, M., Ferrari, M., Long, C. A., Anderson, R., DiFelice, A., and Soummer, R., "High-contrast Imager for Complex Aperture Telescopes (HICAT): II. Design overview and first light results," in [Society of Photo-Optical Instrumentation Engineers (SPIE) Conference Series], Society of Photo-Optical Instrumentation Engineers (SPIE) Conference Series 9143, 914327 (2014).

[57] Floyd, D. J. E., Thomas-Osip, J., and Prieto, G., "Seeing, Wind, and Outer Scale Effects on Image Quality at the Magellan Telescopes," PASP 122, 731-742 (2010).

[58] Ragazzoni, R., "Pupil plane wavefront sensing with an oscillating prism," Journal of Modern Optics 43, 289-293 (1996).

[59] Smith, M. J. and Véran, J.-P., "Implementation of the Altair optimization processes," Proc. SPIE 4839, 964-971 (2003).

[60] Poyneer, L. A. and Véran, J.-P., "Optimal modal Fourier-transform wavefront control," Journal of the Optical Society of America A 22, 1515-1526 (2005).

[61] Soummer, R., Pueyo, L., and Larkin, J., "Detection and Characterization of Exoplanets and Disks Using Projections on Karhunen-Loève Eigenimages," ApJL 755, L28 (2012).

[62] Frazin, R. A., "Utilization of the Wavefront Sensor and Short-exposure Images for Simultaneous Estimation of Quasi-static Aberration and Exoplanet Intensity," ApJ 767, 21 (2013). 